\title{
A multichannel, multiobserver portable event recorder
}

\author{
PETER R. PAMMENT and JOHN B. STEPHENS \\ Animal Behaviour Unit, Psychology Department, University of Queensland \\ Queensland 4067, Australia
}

\begin{abstract}
This paper describes a microprocessor-controlled 64-channel event recorder that supports up to eight remote keyboards utilizing a serial data transmission method. The keyboards can be connected via long twin stereo cable to the encoder unit, which stores the buffered data onto audio cassette tape. This system enables multiple observers to encode multiple events in a field or laboratory setting. The keyboard design is presented in detail.
\end{abstract}

A number of sophisticated electronic observational event recording systems have been developed (Sidowski, 1977). Most can be classified into two basic modes of keyboard entry: (1) parallel systems, able to record simultaneous events, and (2) serial systems, which allow the observer to record only one event at a time. Some of the most popular systems are described by Celhoffer, Boukydis, Minde, and Muir (1977), Fitzpatrick (1977), Stephenson and Roberts (1977), and Torgerson (1977). These and others are compared by Fitzpatrick (1977), who also details the two basic modes of input: "Parallel and serial input modes appear to serve complementary functions. Event timing can be very accurately kept in the parallel mode since the observer has 10 or fewer events (a relatively small number of events) to monitor at a time and he can instantly depress additional keys when additional events occur. When a large number of events are to be studied, the parallel mode works well with multiple passes through videotaped behavioral records. Multiple passes allow accumulation of accurate temporal records on many events but cost additional observation time. A synchronizer signal is used to permit temporal integration of the different passes through the same videotaped record. The parallel input mode is effective with naive observers, requiring only 3 or 4 hours of practice to obtain reasonable reliability with readily observable events. On the other hand, the serial input mode appears optimal in a field setting where trained observers must record a large number of events in a single pass. The gain in number of units per single observation session is offset by a loss in timing accuracy since multiple events occurring simultaneously must be entered one at a time and the observer must also remember to enter the offset of each event. The latter, of course, is an observer problem rather than a hardware limitation" (p. 448).

The use of multiple passes through a videotape does solve the problem of recording a large number of events accurately using a recording device with parallel input mode. However, temporal integration of the different passes is difficult even with a system of synchronizer signals. Videotape stretch and tracking variations can produce time errors of up to $5 \mathrm{sec}$ in a $1 \mathrm{~h}$ recording, and it may be often difficult or impossible to videotape the behavioral events to be recorded.

The use of multiple observers and multiple keyboards can sometimes overcome these problems; each observer records a different set or replicates a standard set of events. In this way, a large number of events can be recorded either in the field or with only one pass through the videotape.

\section{GENERAL DESCRIPTION}

The present system utilizes up to eight remote keyboards, which can be hand held or tabletop mounted, each with eight push-button or toggle switches. These keyboards, along with an audio cassette recorder, are connected to a microprocessor-based controller.

The user assigns specific events to each button and, after selecting a session identification number, starts recording by switching the controller to "run." During the recording session, a crystal-controlled clock interrupts the microprocessor to initiate a scan of each keyboard every $.1 \mathrm{sec}$. If a button has changed state, a 4-byte record of time ( 2 bytes), keyboard identification ( 1 byte), and current button states ( 1 byte) are stored in memory. Thus simultaneous button changes occurring within a keyboard unit are recorded together. There are two data buffers in memory, an input and an output buffer, each of 256 bytes. When the input buffer becomes full, the roles are exchanged. All subsequent incoming data are stored in the new input buffer while the old input buffer (now the output buffer) is stored onto cassette tape. Thus the cassette is only turned on when there is a full buffer of data to be stored. This minimizes the time taken to recover the data at a later date. The data are recorded onto the cassette tape in a binary format similar to the Motorola D2 JBUG, ${ }^{1}$ but with a block identification and checksum scheme.

When the recording session is over, the observer switches the controller unit to "halt" and any data in 


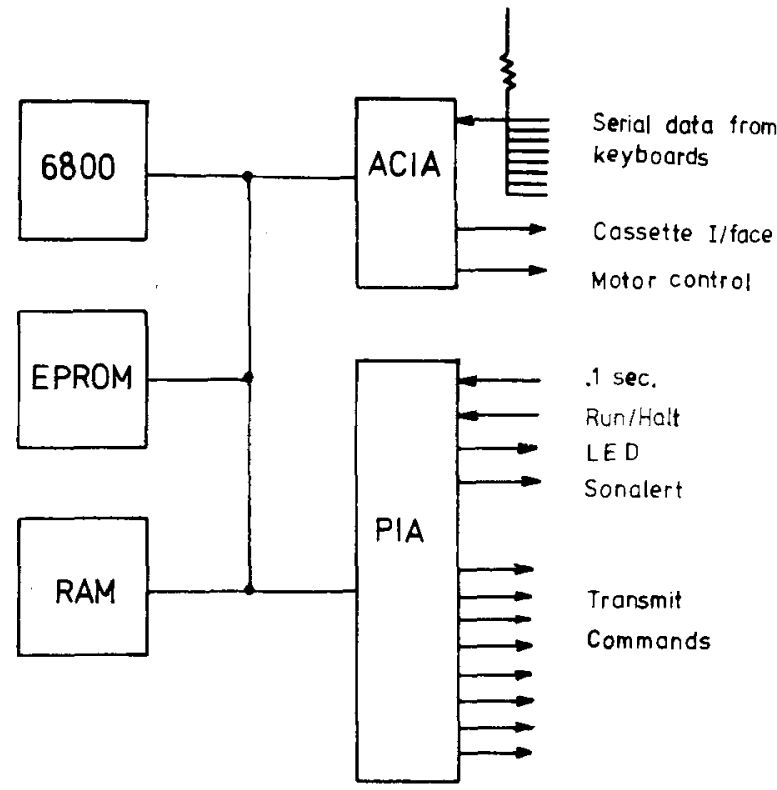

Figure 1. Schematic of the microprocessor event recorder system showing processor, memory, and $\mathbf{I} / 0$ interface devices.

the buffers are output onto the tape. The event recorder has an audio beeper to signal errors, such as a keyboard not responding, or to warn when a $\mathrm{C} 60$ cassette is nearly full.

Figure 1 shows the schematic for the controller. The microprocessor can be any 8-bit general-purpose microprocessor. Our units use the Motorola 6800. Memory requirements are $1 \mathrm{~K}$ of EPROM for control software and $1 \mathrm{~K}$ of $\mathrm{RAM}$ for data buffers and data variables. Input/output interface circuits require an asynchro- nous communication interface adapter (ACIA). The output side is used for the cassette recorder and is standard Kansas City format running at 300 baud. The use of Kansas City format provides a wider choice of computers for decoding, as many commercial microcomputer systems have such tape facilities. The input side of the ACIA is used to receive the data (button states) from each keyboard in a "wired-or" arrangement. A peripheral interface adapter (PIA) is used to read front panel switches, receive clock interrupts, and control the keyboard units via eight output lines.

Each keyboard unit contains a CMOS universal asynchronous receiver transmitter (UART) that is connected by three lines to the controller. One line is the common earth, another carries the serial data, and the third provides both the power and the transmit request. The power and transmit control line is derived from a regulated supply that is adjusted to provide $+5 \mathrm{~V}$ to the UART and its oscillator. This power line is strobed low for about 150 microsec by the PIA control line to signal the UART to transmit a "snapshot" of its button states back to the microprocessor unit. While this line is strobed low, the power supply to the UART is maintained by the capacitor. Figure 2 shows a detailed schematic of the keyboard circuit and power supply. The data as an 8-bit character are transmitted at 1,200 baud. The time to poll all eight keyboards and receive the data is approximately $75 \mathrm{msec}$ (8 characters at 1,200 baud). On start-up, a dummy poll of each keyboard being used clears the UART devices. The use of serial transmission and the combined power and transmit control line makes for simple connection of the keyboards.

The unit has been designed to be portable and can be

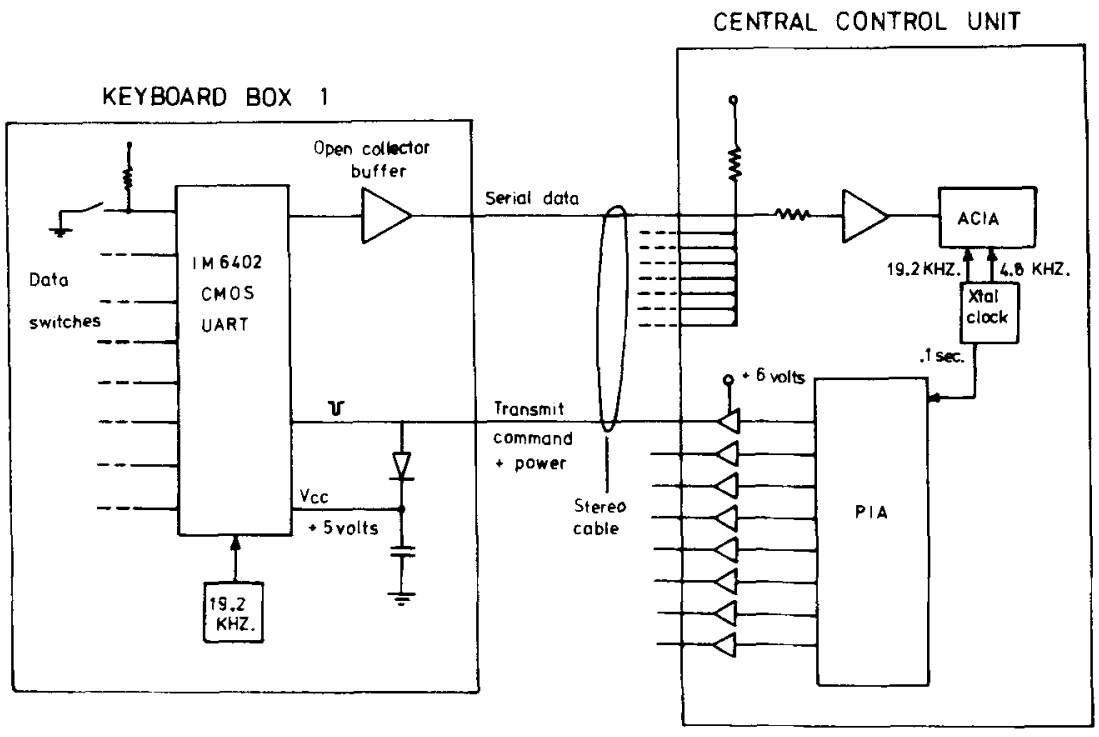

Figure 2. Schematic of the keyboard system, showing keyboard unit design with data, transmit command, and power supply connections to the controller unit. 
powered either by batteries or from normal power outlets.

\section{CosT}

The cost of parts is about $\$ 400$. Labor and assembly time is about $80 \mathrm{~h}$. Further information, including keyboard circuit board design, is available on request. The event recorder control software (M6800 assembly code for a Motorola D1 or D2 kit) or C source code can also be supplied, together with programs to read and decode the data via a Kansas City tape interface on a PDP-11 $1^{2}$ running under $\mathrm{UNIX}^{3}$ (C source) or RSX$11 \mathrm{M}$ (Macro-11; see Footnote 2) systems.

\section{DISCUSSION}

The utility of this event recorder has been demonstrated over the last 3 years by the use of several units to encode animal and human behavioral data (Butler, Beasley, Buckley, \& Endean, 1980; Noller, 1980; Preston, 1980). The recorder has been used by a single observer and by multiple observers to encode videotape and by multiple observers positioned at opposite ends of animal pens. The use of multiple keyboards facilitates field or videotape tests of interobserver reliability. The number of passes through a videotape and the problems of temporal integration can be reduced by the use of multiple observers and a single pass.

This keyboard design can even be used as the basis of a multiple-response unit for laboratory experimentation with eight subjects being run simultaneously. The basic design can be implemented on any commercial microprocessor system that has a serial input port and a parallel output port with a bit for each keyboard to be controlled.

\section{REFERENCES}

Butler, J. E., Beasley, W. F., Buckley, D., \& Endean, L. Pupil task involvement in secondary science classrooms. Research in Science Education, 1980, 10, 93-106.

Celhoffer, L., Boukydis, C., Minde, K., \& Muir, E. The DCR-II event recorder: A portable high-speed digital cassette system with direct computer access. Behavior Research Methods \& Instrumentation, 1977, 9, 442-446.

Fitzpatrick, L. J. Automated data collection for observed events. Behavior Research Methods \& Instrumentation, 1977, 9, 447-451.

Nollen, P. Cross-gender effect in two-child families. Developmental Psychology, 1980, 16, 159-160.

Preston, A. P. Behaviour of laying hens on a controlling feeding schedule. 6th European Poultry Conference (Vol. 4) (Hamburg), September 1980, pp. 191-198.

Sidowski, J. B. Observational research: Some instrumented systems for scoring and storing behavioral data. Behavior Research Methods \& Instrumentation, 1977, 9, 403-404.

Stephenson, G. R., \& Roberts, T. W. The SSR System 7: A general encoding system with computerized transcription. Behavior Research Methods \& Instrumentation, 1977, 9, 434-441.

Torgerson, L. Datamyte 900. Behavior Research Methods \& Instrumentation, 1977, 9, 405-406.

\section{NOTES}

1. JBUG is a trademark of the Motorola Corporation.

2. PDP-11, RSX-11M, and MACRO-11 are trademarks of the Digital Equipment Corporation.

3. UNIX is a trademark of the Western Electric Corporation.

(Received for publication October 23, 1981; accepted November 4,1981 .) 